$(200)$

R290

no. $85-165$

WATER-QUALITY DATA FOR 34 SITES,

APRIL AND JUNE 1984, NEAR THE Y-12 PLANT,

THE OAK RIDGE RESERVATION, TENNESSEE
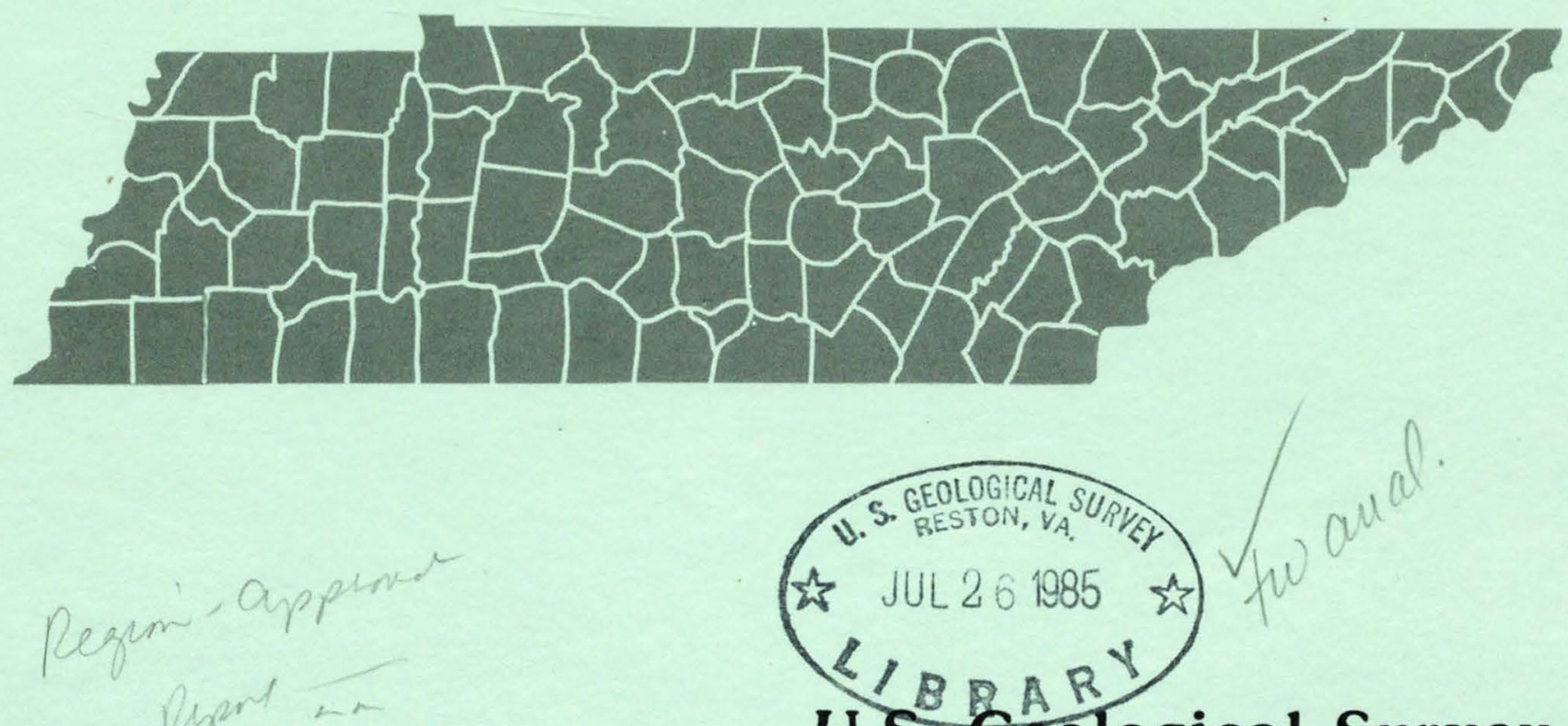

U.S. Geological Survey

Open-File Report 85-165

Prepared in cooperation with the

U.S. Department of Energy 
WATER-QUALITY DATA FOR 34 SITES, APRIL AND JUNE 1984, NEAR THE Y-12 PLANT, THE OAK RIDGE RESERVATION, TENNESSEE Pamela J. Pulliam

U.S. GEOLOGICAL SURVEY

Open-File Report 85-165

\section{Open-fie re ( il \\ Geological Survey \\ (U.S.)}

Prepared in cooperation with the

U.S. DEPARTMENT OF ENERGY 


\section{UNITED STATES DEPARTMENT OF THE INTERIOR \\ DONALD PAUL HODEL, Secretary \\ GEOLOGICAL SURVEY}

Dallas L. Peck, Director

For additional information write to:

District Chief

U.S. Geological Survey

A-413 Federal Building

U.S. Courthouse

Nashvil1e, Tennessee 37203
Copies of this report can be purchased from:

Open-File Services Section Western Distribution Branch U.S. Geological Survey Box 25425, Federal Center Lakewood, Colorado 80225 ( Telephone: (303) 236-7476) 


\section{CONTENTS}

Abstract 1

In troduction

Description of the area 4

Approach 4

Explanation of data 6

References

13

\section{ILLUSTRATIONS}

Figure 1. Map showing study area 2

2. Map showing sampling sites

\section{3}

\section{TABLES}

Table 1. Sampling locations $\mathbf{5}$

2. Water-quality parameters determined 6

3. Field analyses 7

4. Major constituents and properties $\mathbf{9}$

5. Trace constituents and compounds $\mathbf{1 0}$

6. Radiochemicals 12

FACTORS FOR CONVERTING INCH-POUND UNITS TO INTERNATIONAL SYSTEM OF UNITS (SI)

\section{Mu1tiply}

cubic foot per second $\left(\mathrm{ft}^{3} / \mathrm{s}\right)$

foot $(\mathrm{ft})$

acre

square mile $\left(\mathrm{mi}^{2}\right)$

mile (mi)

picocuries ( $p C i)$ byy

To obtain
0.02832 cubic meter per second $\left(\mathrm{m}^{3} / \mathrm{s}\right)$

0.3048 meter $(\mathrm{m})$

0.4047 square hectometer $\left(\mathrm{hm}^{2}\right)$

2.590 square kilometer $\left(\mathrm{km}^{2}\right)$

1.609 kilometer $(\mathrm{km})$

27.0

disintegrations per second 



\title{
WATER-QUALITY DATA FOR 34 SITES, APRIL AND JUNE 1984, NEAR THE Y-12 PLANT, THE OAK RIDGE RESERVATION, TENNESSEE
}

\author{
Pamela J. Pulliam
}

\begin{abstract}
Water-quality data were collected at 34 sites in the vicinity of the Y-12 Plant, Oak Ridge, Tennessee, on April 12 and 13, 1984. Concentrations of dissolved major and trace constituents were determined; field determinations of specific conductance, $\mathrm{pH}$, temperature, alkalinity, and dissolved oxygen were made. Gross alpha and beta activity were determined for 10 of the 34 sites sampled in April, and concentrations of dissolved organic carbon and oil and grease were determined for 11 sites sampled on June 3, 1984.
\end{abstract}

\section{INTRODUCTION}

Water-quality data were collected on Apri1 12 and 13, 1984 , during high base flow by the U.S. Geological Survey in watersheds near the Y-12 Plant at the Oak Ridge Reservation, Tenn. (fig. 1). The watersheds range in size from 0.33 to $5.92 \mathrm{mi}{ }^{2}$. Concentrations of dissolved major and trace constituents were determined; field determinations of specific conductance, $\mathrm{pH}$, temperature, alkalinity, and dissolved oxygen were made. This data collection is part of a study done in cooperation with the Department of Energy. The purpose of the study is to describe the regional ground-water flow system and to determine the extent of any effects on ground-water quality resulting from activities at the Y-12 P1 ant.

The major sources of contamination in the vicinity of the Y-12 Plant are the S-3 ponds, located at the headwaters of Bear Creek at the eastern end of the valley, and the Bear Creek burial grounds and oil landfarm, located north of Bear Creek, southwest of the S-3 ponds (fig. 2). Because a significant part of the flow in the streams is comprised of ground water, determining the quality of water from these streams may indicate the extent of ground-water contamination and the direction of regional groundwater flow. 


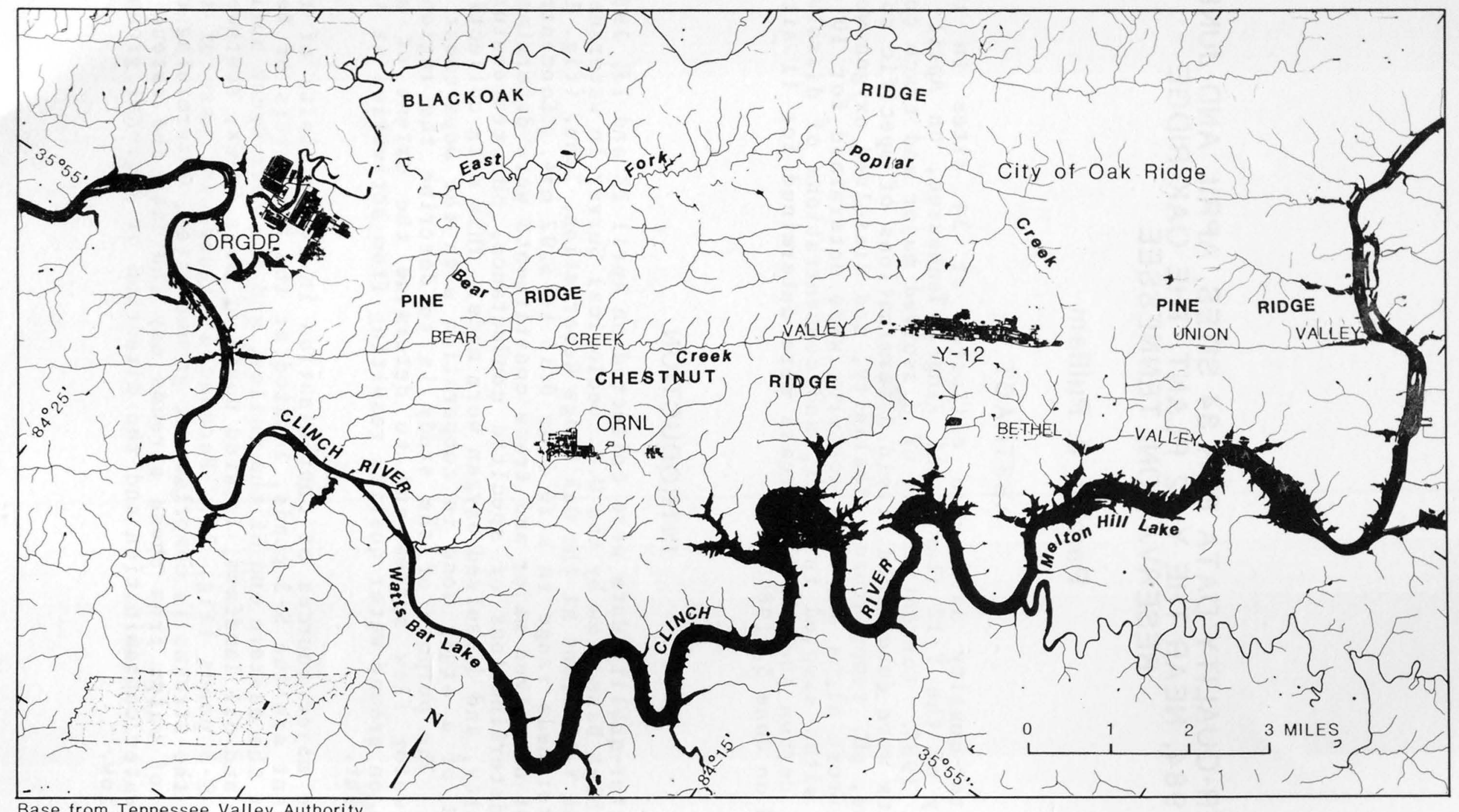

Base from Tennessee Valley Authority

1:24,000 map S-16A, revised in part June 1974

Figure 1.--Study area. 


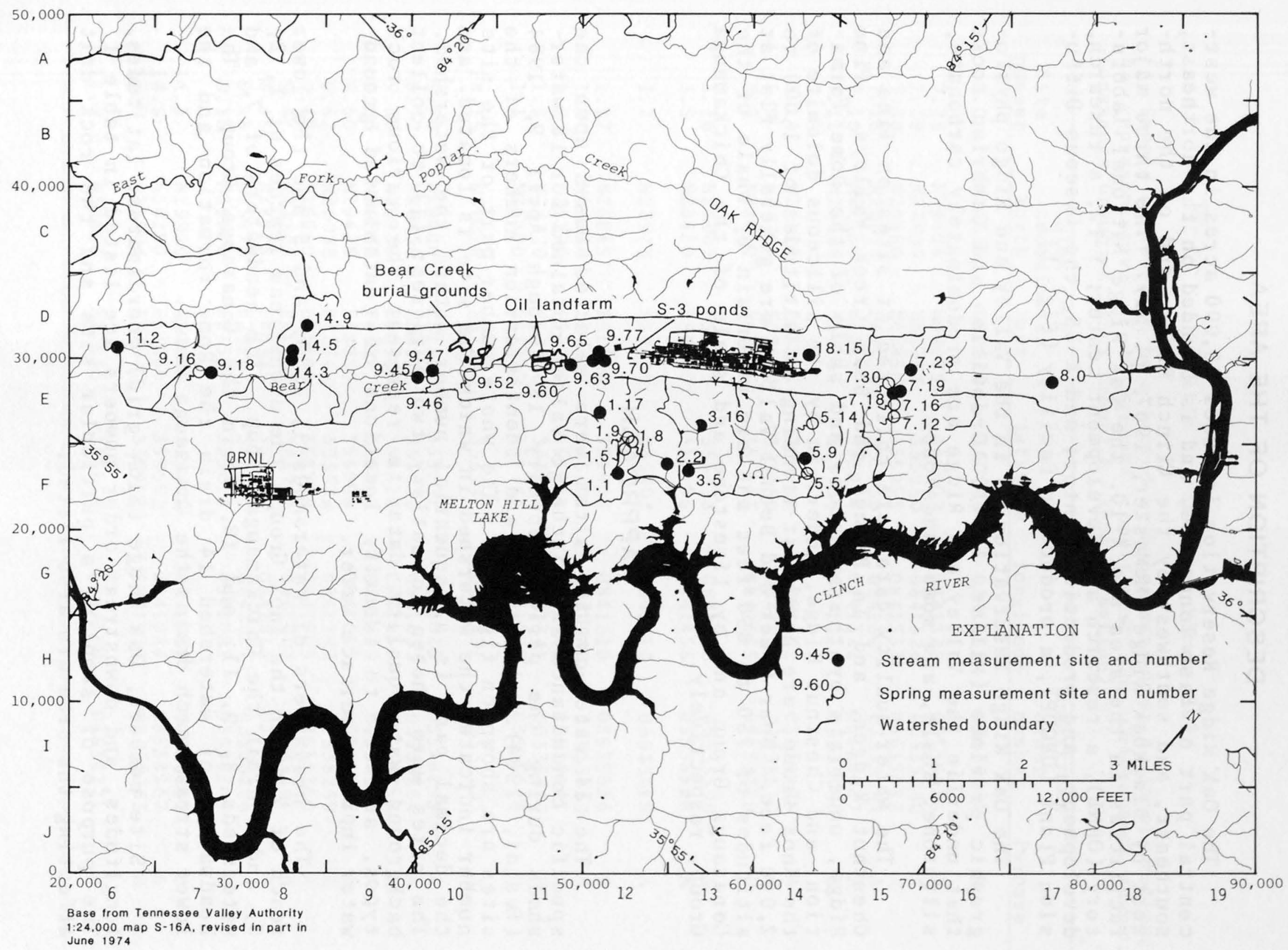

Figure 2.--Sampling sites. 


\section{DESCRIPTION OF THE AREA}

The Oak Ridge Reservation includes 58,000 acres in the westcentral part of East Tennessee, and is bounded on the northeast, southeast, and southwest by the $\mathrm{Cl}$ inch River, and on the northwest by B1ackoak Ridge (McMaster, 1967, p. N2). The three major facilities in the area are $X-10$, the Oak Ridge National Laboratory (ORNL), a research and development center; Y-12, a research, development, and production center; and K-25, the Gaseous Diffusion Plant (ORGDP), a production facility (fig. 1).

The Oak Ridge Reservation is in the Valley and Ridge physiographic province (Miller, 1974). Ordovician and Cambrian rocks that underlie the Valley and Ridge are predominately carbonate, siltstone, shale, and some sandstone.

The water-quality data were collected at sites on Pine and Chestnut Ridges, and in Bethel and Bear Creek Valleys. Pine Ridge, underlain by the sandstone and shale of the Rome Formation, and Chestnut Ridge, underlain by the siliceous dolomite of the Knox Group, are northeast-trending at altitudes of 1,000 to $2,000 \mathrm{feet}$. Bear Creek and Bethel Valleys are generally flat at altitudes of 750 to 850 feet and are underlain by shale of the Conasauga Group and by 1 imestone and shale of the Chickamauga Group, respectively.

\section{APPROACH}

The 34 water-quality sites were selected based upon the specific conductance and discharge values obtained for 18 watersheds during the period February 15 through April 9, 1984 (Evaldi, 1984). Locations and identification numbers of the sites are shown on figure 2. The whole number part of the site number indicates the watershed in which the site is located, and the decimal part is a sequential number within the watershed. The sites were chosen to satisfy two purposes: (1) to collect background water-quality data to represent the various rock types, and (2) to identify locations of contaminated groundwater input to surface water.

The headwaters of watersheds $1,2,3$, and 5 and the lower reach of 7 drain the Knox Group, the downstream reaches of 1,2 , 3 , and 5 drain the Chickamauga Group. The headwaters of 7 , and watersheds $8,9,11$, and 18, drain the Conasauga Group. The headwaters of watershed 14 drain the Rome Formation and the lower stream reach drains the Conasauga Group.

Site numbers, Oak Ridge (S16A grid) coordinates, latitudes, longitudes, and downstream order numbers are listed in table 1 . The purpose of sampling a particular site and the rock unit affecting the site are also listed. 
Table 1.--Sampling locations

[A, Sample believed to be affected by human activity; B, Sample believed to be representative of background conditions; $\mathrm{CH}$, Chickamauga Group; $\mathrm{CO}$, Conasauga Group; K, Knox Group; R, Rome Formation]

\begin{tabular}{|c|c|c|c|c|c|c|c|}
\hline & SI6A & rid & & & wnstream & & \\
\hline $\begin{array}{c}\text { Site } \\
\text { number }\end{array}$ & $\begin{array}{l}\text { coord } \\
\text { North }\end{array}$ & $\begin{array}{l}\text { ates } \\
\text { East }\end{array}$ & Latitude & Long itude & $\begin{array}{l}\text { order } \\
\text { number }\end{array}$ & Purpose & $\begin{array}{l}\text { Rock } \\
\text { unit }\end{array}$ \\
\hline 8.0 & N28580 & E7 7420 & 360046 & 0841157 & 03534880 & A & $\mathrm{CO}$ \\
\hline $\begin{array}{l}7.23 \\
7.19 \\
7.30 \\
7.18 \\
7.16 \\
7.12\end{array}$ & $\begin{array}{l}\text { N29405 } \\
\text { N2 } 8055 \\
\text { N28550 } \\
\text { N2 } 8050 \\
\text { N27320 } \\
\text { N2 } 6580\end{array}$ & $\begin{array}{l}\text { E69175 } \\
\text { E68285 } \\
\text { E67850 } \\
\text { E68125 } \\
\text { E68177 } \\
\text { E68153 }\end{array}$ & $\begin{array}{l}360007 \\
355950 \\
355953 \\
355950 \\
355944 \\
355938\end{array}$ & $\begin{array}{l}0841325 \\
0841326 \\
0841333 \\
0841327 \\
0841322 \\
0841316\end{array}$ & $\begin{array}{l}03535076 \\
03535080 \\
03535082 \\
03535084 \\
03535087 \\
03535090\end{array}$ & $\begin{array}{l}\text { B } \\
\text { A } \\
\text { A } \\
\text { A } \\
\text { B } \\
\text { B }\end{array}$ & $\begin{array}{r}\mathrm{CO} \\
\mathrm{CO} \\
\mathrm{CO} \\
\mathrm{CO} \\
\mathrm{K} \\
\mathrm{K}\end{array}$ \\
\hline $\begin{array}{l}5.14 \\
5.9 \\
5.5\end{array}$ & $\begin{array}{l}\text { N2 } 6175 \\
\text { N24205 } \\
\text { N23310 }\end{array}$ & $\begin{array}{l}\text { E63375 } \\
\text { E62940 } \\
\text { E63095 }\end{array}$ & $\begin{array}{l}355909 \\
355850 \\
355843\end{array}$ & $\begin{array}{l}0841402 \\
0841353 \\
0841345\end{array}$ & $\begin{array}{l}03535105 \\
03535110 \\
03535120\end{array}$ & $\begin{array}{l}\text { A } \\
\text { A } \\
\text { B }\end{array}$ & $\begin{array}{c}\mathrm{K} \\
\mathrm{CH}, \mathrm{K} \\
\mathrm{CH}\end{array}$ \\
\hline $\begin{array}{l}3.16 \\
3.5\end{array}$ & $\begin{array}{l}\text { N2 } 6065 \\
\text { N23420 }\end{array}$ & $\begin{array}{l}\text { E5 } 6875 \\
\text { E5 6180 }\end{array}$ & $\begin{array}{l}355853 \\
355805\end{array}$ & $\begin{array}{l}0841508 \\
0841455\end{array}$ & $\begin{array}{l}03535590 \\
03535598\end{array}$ & $\begin{array}{l}\mathrm{A} \\
\mathrm{A}\end{array}$ & $\begin{array}{r}\mathrm{K} \\
\mathrm{CH}\end{array}$ \\
\hline 2.2 & N23925 & E5 4880 & 355802 & 0841512 & 03535615 & A & $\mathrm{CH}$ \\
\hline $\begin{array}{l}1.17 \\
1.9 \\
1.8 \\
1.5 \\
1.1\end{array}$ & $\begin{array}{l}\text { N2 } 6845 \\
\text { N2 } 5280 \\
\text { N2 } 5170 \\
\text { N2 } 4720 \\
\text { N23300 }\end{array}$ & $\begin{array}{l}\text { E51100 } \\
\text { E5 2645 } \\
\text { E52775 } \\
\text { E5 2370 } \\
\text { E5 2025 }\end{array}$ & $\begin{array}{l}355805 \\
355802 \\
355801 \\
355756 \\
355741\end{array}$ & $\begin{array}{l}0841610 \\
0841543 \\
0841541 \\
0841541 \\
0841537\end{array}$ & $\begin{array}{l}03535636 \\
03535639 \\
03535641 \\
03535643 \\
03535648\end{array}$ & $\begin{array}{l}B \\
B \\
A \\
B \\
B\end{array}$ & $\begin{array}{l}\mathrm{K} \\
\mathrm{K} \\
\mathrm{K} \\
\mathrm{K}, \mathrm{CH} \\
\mathrm{CH}\end{array}$ \\
\hline 18.15 & N30218 & E6 3200 & 355941 & 0841431 & 03538233 & A & $\mathrm{CO}$ \\
\hline $\begin{array}{l}9.70 \\
9.77 \\
9.65 \\
9.63 \\
9.60 \\
9.52 \\
9.47 \\
9.46 \\
9.45 \\
9.18 \\
9.16\end{array}$ & $\begin{array}{l}\text { N29906 } \\
\text { N3 } 0450 \\
\text { N29920 } \\
\text { N29583 } \\
\text { N29425 } \\
\text { N29038 } \\
\text { N28940 } \\
\text { N28805 } \\
\text { N28925 } \\
\text { N29175 } \\
\text { N29180 }\end{array}$ & $\begin{array}{l}\text { E51238 } \\
\text { E50918 } \\
\text { E50560 } \\
\text { E4 9117 } \\
\text { E4 8085 } \\
\text { E4 3342 } \\
\text { E41240 } \\
\text { E41168 } \\
\text { E40315 } \\
\text { E2 8167 } \\
\text { E27550 }\end{array}$ & $\begin{array}{l}355831 \\
355836 \\
355828 \\
355817 \\
355808 \\
355739 \\
355728 \\
355726 \\
355722 \\
355616 \\
355614\end{array}$ & $\begin{array}{l}0841629 \\
0841635 \\
0841637 \\
0841649 \\
0841653 \\
0841742 \\
0841803 \\
0841803 \\
0841813 \\
0842017 \\
0842022\end{array}$ & $\begin{array}{l}03538253 \\
03538254 \\
03538255 \\
03538256 \\
03538257 \\
03538259 \\
03538260 \\
03538261 \\
03538262 \\
03538268 \\
03538269\end{array}$ & $\begin{array}{l}\text { A } \\
\text { A } \\
\text { A } \\
\text { A } \\
\text { B } \\
\text { A } \\
\text { A } \\
\text { B } \\
\text { A } \\
\text { A } \\
\text { B }\end{array}$ & $\begin{array}{l}\text { CO } \\
\text { CO } \\
\text { CO } \\
\text { CO } \\
\text { CO } \\
\text { CO } \\
\text { CO } \\
\text { CO } \\
\text { CO } \\
\text { CO } \\
\text { CO }\end{array}$ \\
\hline $\begin{array}{l}14.3 \\
14.5 \\
14.9\end{array}$ & $\begin{array}{l}\text { N29920 } \\
\text { N30250 } \\
\text { N31906 }\end{array}$ & $\begin{array}{l}\text { E3 } 3043 \\
\text { E33060 } \\
\text { E33943 }\end{array}$ & $\begin{array}{l}355649 \\
355652 \\
355711\end{array}$ & $\begin{array}{l}0841932 \\
0841934 \\
0841937\end{array}$ & $\begin{array}{l}03538266 \\
03538267 \\
03538265\end{array}$ & $\begin{array}{l}\mathrm{B} \\
\mathrm{B} \\
\mathrm{B}\end{array}$ & $\begin{array}{r}\mathrm{CO} \\
\mathrm{CO} \\
\mathrm{R}\end{array}$ \\
\hline 11.2 & N30650 & E22846 & 355558 & 0842120 & 03538271 & B & $\mathrm{CO}$ \\
\hline
\end{tabular}




\section{EXPLANATION OF DATA}

A listing of the water-quality parameters collected in the vicinity of the Y-12 facility is shown in table 2. The samples collected on April 12 and 13 were analyzed for all the 1 isted parameters with the exception of dissolved organic carbon and oil and grease, which were obtained on June 3, 1984.

Table 2. - Water-quality parameters determined

Fi eld:

Water temperature

Specific conductance $\mathrm{pH}$
Al ka1inity

Dissolved oxygen

La boratory:

Major constituents (dissolved) or properties:

Calcium

Magnesium

Sodium

Potassium

Alkalinity
Chloride

Sulfate

Nitrogen

Phosphorus

Residue on evaporation

Trace constituents (total recoverable) and compounds:

$\begin{array}{llll}\text { Barium } & \text { Copper } & \text { Lithium } & \text { Strontium } \\ \text { Beryliium } & \text { Cyanide } & \text { Manganese } & \text { Uranium } \\ \text { Cadmium } & \text { Iron } & \text { Mercury } & \text { Vanadium } \\ \text { Chromium } & \text { Lead } & \text { Molybdenum } & \text { Zinc } \\ \text { Cobalt } & & & \end{array}$

Other parameters:

Gross alpha (dissolved and suspended)

Gross beta (dissolved, total, and suspended)

Dissolved and total organic carbon

Oil and grease

Field Analyses: Measurements of temperature, specific conductance, $\mathrm{pH}$, dissolved oxygen, alkalinity, and streamflow were made at each site at the time of sample collection (table 3). In some cases insufficient flow prevented measurement of discharge and (or) dissolved oxygen. Temperature, specific conductance, and discharge were determined on June 3. 
Table 3.--Field analyses

\begin{tabular}{|c|c|c|c|c|c|c|c|}
\hline $\begin{array}{c}\text { Site } \\
\text { number }\end{array}$ & Date & $\begin{array}{c}\text { Temper- } \\
\text { a ture } \\
\left(\begin{array}{cc}0 & C\end{array}\right)\end{array}$ & $\begin{array}{l}\text { Spec- } \\
\text { cific } \\
\text { con- } \\
\text { duct- } \\
\text { ance } \\
(\mu S / c m)\end{array}$ & $\mathrm{pH}$ & $\begin{array}{l}\text { Alka- } \\
1 \text { inity } \\
\text { field } \\
(\mathrm{mg} / \mathrm{L} \\
\text { as } \\
\mathrm{CaCO}_{3} \text { ) } \\
\end{array}$ & $\begin{array}{l}\text { Oxygen, } \\
\text { dis- } \\
\text { solved } \\
(\mathrm{mg} / \mathrm{L})\end{array}$ & $\begin{array}{c}\text { Dis- } \\
\text { charge } \\
\text { instan- } \\
\text { taneous } \\
\left(\mathrm{ft} \mathrm{t}^{3} / \mathrm{s}\right) \\
\end{array}$ \\
\hline 8.0 & $4 / 12 / 84$ & 13.0 & 318 & 7.1 & 128 & -- & -- \\
\hline $\begin{array}{l}7.23 \\
7.19 \\
7.30 \\
7.18 \\
7.16 \\
7.12\end{array}$ & $\begin{array}{l}4 / 12 / 84 \\
6 / 3 / 84 \\
4 / 12 / 84 \\
6 / 3 / 84 \\
4 / 12 / 84 \\
4 / 12 / 84 \\
6 / 3 / 84 \\
4 / 12 / 84 \\
4 / 12 / 84\end{array}$ & $\begin{array}{l}10.0 \\
17.0 \\
15.0 \\
16.0 \\
12.5 \\
14.0 \\
15.5 \\
15.0 \\
14.0\end{array}$ & $\begin{array}{r}85 \\
105 \\
460 \\
660 \\
400 \\
380 \\
425 \\
282 \\
232\end{array}$ & $\begin{array}{r}6.0 \\
-- \\
7.3 \\
-. \\
7.1 \\
7.5 \\
-.- \\
7.4 \\
7.4\end{array}$ & $\begin{array}{r}-- \\
-- \\
198 \\
-- \\
198 \\
186 \\
-- \\
156 \\
140\end{array}$ & $\begin{array}{r}9.2 \\
-- \\
7.0 \\
-- \\
5.5 \\
10.4 \\
-- \\
8.2 \\
7.2\end{array}$ & $\begin{array}{l}0.05 \\
.01 \\
.19 \\
.07 \\
.07 \\
.25 \\
.22 \\
.32 \\
.19\end{array}$ \\
\hline $\begin{array}{l}5.14 \\
5.9 \\
5.5\end{array}$ & $\begin{array}{l}4 / 12 / 84 \\
4 / 12 / 84 \\
4 / 13 / 84\end{array}$ & $\begin{array}{l}13.0 \\
14.0 \\
14.0\end{array}$ & $\begin{array}{l}183 \\
262 \\
245\end{array}$ & $\begin{array}{l}7.0 \\
8.0 \\
7.4\end{array}$ & $\begin{array}{c}-- \\
100\end{array}$ & $\begin{array}{l}7.7 \\
8.2 \\
8.0\end{array}$ & $\begin{array}{l}.16 \\
.88 \\
.13\end{array}$ \\
\hline $\begin{array}{l}3.16 \\
3.5\end{array}$ & $\begin{array}{l}4 / 12 / 84 \\
4 / 12 / 84\end{array}$ & $\begin{array}{l}12.5 \\
12.0\end{array}$ & $\begin{array}{l}360 \\
324\end{array}$ & $\begin{array}{l}7.2 \\
8.7\end{array}$ & -- & $12 . \overline{6}$ & $\begin{array}{l}.27 \\
3.1\end{array}$ \\
\hline $\begin{array}{l}2.2 \\
1.17 \\
1.9 \\
1.8 \\
1.5 \\
1.1\end{array}$ & $\begin{array}{l}4 / 13 / 84 \\
6 / 3 / 84 \\
4 / 12 / 84 \\
4 / 12 / 84 \\
4 / 12 / 84 \\
4 / 13 / 84 \\
4 / 12 / 84\end{array}$ & $\begin{array}{l}16.5 \\
20.0 \\
15.0 \\
12.0 \\
12.0 \\
12.5 \\
14.5\end{array}$ & $\begin{array}{l}200 \\
225 \\
115 \\
158 \\
240 \\
225 \\
190\end{array}$ & $\begin{array}{l}8.0 \\
-- \\
7.3 \\
6.7 \\
6.7 \\
6.8 \\
7.7\end{array}$ & $\begin{array}{r}96 \\
-- \\
54 \\
77 \\
111 \\
111 \\
94\end{array}$ & $\begin{array}{r}9.0 \\
-. \\
8.9 \\
9.2 \\
7.9 \\
8.2 \\
10.2\end{array}$ & $\begin{array}{l}<.01 \\
.01 \\
.14 \\
.47 \\
.02 \\
.37 \\
.87\end{array}$ \\
\hline 18.15 & $\begin{array}{l}4 / 12 / 84 \\
6 / 3 / 84\end{array}$ & $\begin{array}{l}15.5 \\
18.5\end{array}$ & $\begin{array}{l}780 \\
800\end{array}$ & $\begin{array}{r}8.0 \\
--\end{array}$ & -- & $\begin{array}{r}13.4 \\
--\end{array}$ & $\begin{array}{r}.00 \\
<.01\end{array}$ \\
\hline 9.70 & $\begin{array}{l}4 / 13 / 84 \\
6 / 3 / 84\end{array}$ & $\begin{array}{l}11.0 \\
15.0\end{array}$ & $\begin{array}{l}4,000 \\
3,900\end{array}$ & 6.6 & -- & 1.6 & $\begin{array}{r}.02 \\
<.01\end{array}$ \\
\hline 9.77 & $\begin{array}{l}4 / 13 / 84 \\
6 / 3 / 84\end{array}$ & $\begin{array}{l}14.0 \\
21.0\end{array}$ & $\begin{array}{r}86 \\
145\end{array}$ & $\begin{array}{r}6.8 \\
--\end{array}$ & -- & 9.2 & $\begin{array}{l}.08 \\
.01\end{array}$ \\
\hline $\begin{array}{l}9.65 \\
9.63\end{array}$ & $\begin{array}{l}4 / 13 / 84 \\
4 / 13 / 84 \\
6 / 3 / 84\end{array}$ & $\begin{array}{l}13.0 \\
17.0 \\
22.0\end{array}$ & $\begin{array}{l}2,300 \\
1,800 \\
2,600\end{array}$ & $\begin{array}{r}5.9 \\
7.6 \\
--\end{array}$ & $\begin{array}{l}-- \\
--\end{array}$ & $\begin{array}{r}8.9 \\
9.0 \\
-.\end{array}$ & $\begin{array}{l}.11 \\
.29 \\
.07\end{array}$ \\
\hline $\begin{array}{l}9.60 \\
9.52 \\
9.47\end{array}$ & $\begin{array}{l}4 / 13 / 84 \\
4 / 12 / 84 \\
4 / 12 / 84 \\
6 / 3 / 84\end{array}$ & $\begin{array}{l}14.0 \\
13.0 \\
16.0 \\
16.0\end{array}$ & $\begin{array}{l}240 \\
530 \\
540 \\
780\end{array}$ & $\begin{array}{r}7.5 \\
7.2 \\
8.1 \\
-.-\end{array}$ & $\begin{array}{l}-- \\
-- \\
--\end{array}$ & $\begin{array}{r}9.0 \\
4.3 \\
10.4 \\
.-\end{array}$ & $\begin{array}{r}.14 \\
.21 \\
1.3 \\
.13\end{array}$ \\
\hline $\begin{array}{l}9.46 \\
9.45 \\
9.18 \\
9.16\end{array}$ & $\begin{array}{l}4 / 12 / 84 \\
4 / 12 / 84 \\
4 / 12 / 84 \\
6 / 3 / 84 \\
4 / 12 / 84\end{array}$ & $\begin{array}{l}14.0 \\
11.0 \\
15.0 \\
14.5 \\
12.0\end{array}$ & $\begin{array}{l}360 \\
480 \\
320 \\
420 \\
221\end{array}$ & $\begin{array}{r}7.2 \\
7.7 \\
8.5 \\
-.- \\
7.3\end{array}$ & $\begin{array}{l}-- \\
-- \\
-- \\
--\end{array}$ & $\begin{array}{r}6.6 \\
10.2 \\
10.0 \\
-. \\
9.6\end{array}$ & $\begin{array}{l}.75 \\
1.9 \\
4.3 \\
1.2 \\
.71\end{array}$ \\
\hline $\begin{array}{l}14.3 \\
14.5 \\
14.9\end{array}$ & $\begin{array}{l}4 / 12 / 84 \\
6 / 3 / 84 \\
4 / 12 / 84 \\
4 / 12 / 84\end{array}$ & $\begin{array}{r}16.0 \\
14.0 \\
11.0 \\
9.5\end{array}$ & $\begin{array}{r}68 \\
123 \\
47 \\
48\end{array}$ & $\begin{array}{r}7.8 \\
-.- \\
9.2 \\
8.3\end{array}$ & $\begin{array}{l}-- \\
-- \\
--\end{array}$ & $\begin{array}{r}10.5 \\
-. \\
9.8 \\
10.0\end{array}$ & $\begin{array}{l}.21 \\
.04 \\
.03 \\
.10\end{array}$ \\
\hline 11.2 & $4 / 12 / 84$ & 13.0 & 35 & 6.0 & 7 & 7.8 & .01 \\
\hline
\end{tabular}


Laboratory Analyses: The water-quality data collected during April and June are listed in table 4. Samples were filtered through a 0.45 micrometer membrane filter at the time of collection. Trace constituent and oil and grease concentrations were determined from unfiltered samples (table 5). These analyses were done by the U.S. Geological Survey Central Laboratory, Doraville, Georgia, using methods described in skougstad and others (1979) and Goerlitz and Brown (1972). The radionuclides (table 6) were determined at the Geological Survey Central Laboratory in Denver, Colorado, using methods described in Thacker and others (1977). 
Table 4.--Major constituents and properties

\begin{tabular}{|c|c|c|c|c|c|c|c|c|c|c|c|}
\hline $\begin{array}{c}\text { Site } \\
\text { number }\end{array}$ & $\begin{array}{c}\text { Date } \\
\text { of } \\
\text { sample }\end{array}$ & $\begin{array}{l}\text { Calcium } \\
\text { dis- } \\
\text { solved } \\
\text { (mg/L } \\
\text { as Ca) }\end{array}$ & $\begin{array}{l}\text { Magne- } \\
\text { sium, } \\
\text { dis- } \\
\text { solved } \\
\text { (mg/L } \\
\text { as } \mathrm{Mg} \text { ) }\end{array}$ & $\begin{array}{l}\text { Sodium, } \\
\text { dis- } \\
\text { solved } \\
\text { (mg/L } \\
\text { as } \mathrm{Na}) \\
\end{array}$ & $\begin{array}{l}\text { Potas- } \\
\text { sium, } \\
\text { dis- } \\
\text { solved } \\
\text { (mg/L } \\
\text { as K) }\end{array}$ & $\begin{array}{l}\text { Alka- } \\
1 \text { inity } \\
1 \mathrm{ab} \\
(\mathrm{mg} / \mathrm{L} \\
\text { as } \\
\mathrm{CaCO} \text { ) }\end{array}$ & $\begin{array}{l}\text { Chlo- } \\
\text { ride, } \\
\text { dis- } \\
\text { solved } \\
\text { (mg/L } \\
\text { as Cl) }\end{array}$ & $\begin{array}{l}\text { Sulfate } \\
\text { dis- } \\
\text { solved } \\
(\mathrm{mg} / \mathrm{L} \\
\left.\text { as } \mathrm{SO}_{4}\right) \\
\end{array}$ & $\begin{array}{l}\text { Nitro- } \\
\text { gen, } \\
\mathrm{NO}^{2}+\mathrm{NO}^{\circ} \\
\text { di s- } \\
\text { solved } \\
\text { (mg/L } \\
\text { as N) }\end{array}$ & $\begin{array}{l}\text { Phos- } \\
\text { phorus, } \\
\text { di s- } \\
\text { solved } \\
\text { (mg/L } \\
\text { as P) }\end{array}$ & $\begin{array}{l}\text { Solids } \\
\text { residue } \\
\text { at } 180^{\circ} \mathrm{C} \\
\text { dis- } \\
\text { solved } \\
(\mathrm{mg} / \mathrm{L}) \\
\end{array}$ \\
\hline 8.0 & $4 / 12 / 84$ & 43 & 11 & 3.3 & 1.7 & 122 & 2.5 & 43 & .560 & $<.010$ & 173 \\
\hline $\begin{array}{l}7.23 \\
7.19 \\
7.30 \\
7.18 \\
7.16 \\
7.12\end{array}$ & $\begin{array}{l}4 / 12 / 84 \\
4 / 12 / 84 \\
4 / 12 / 84 \\
4 / 12 / 84 \\
4 / 12 / 84 \\
4 / 12 / 84\end{array}$ & $\begin{array}{l}8.6 \\
41 \\
70 \\
56 \\
31 \\
28\end{array}$ & $\begin{array}{l}2.7 \\
15 \\
6.7 \\
11 \\
18 \\
16\end{array}$ & $\begin{array}{c}2.5 \\
15 \\
2.6 \\
4.6 \\
.90 \\
.70\end{array}$ & $\begin{array}{l}1.5 \\
9.6 \\
1.5 \\
1.6 \\
.60 \\
.70\end{array}$ & $\begin{array}{r}31 \\
198 \\
194 \\
179 \\
156 \\
139\end{array}$ & $\begin{array}{c}1.3 \\
24 \\
4.1 \\
9.4 \\
2.0 \\
1.6\end{array}$ & $\begin{array}{l}11 \\
8.9 \\
17 \\
16 \\
4.0 \\
2.2\end{array}$ & $\begin{array}{l}<.100 \\
.190 \\
.950 \\
.690 \\
.320 \\
.170\end{array}$ & $\begin{array}{l}<.010 \\
<.010 \\
<.010 \\
<.010 \\
<.010 \\
<.010\end{array}$ & $\begin{array}{r}64 \\
270 \\
256 \\
231 \\
184 \\
159\end{array}$ \\
\hline $\begin{array}{l}5.14 \\
5.9 \\
5.5\end{array}$ & $\begin{array}{l}4 / 12 / 84 \\
4 / 12 / 84 \\
4 / 13 / 84\end{array}$ & $\begin{array}{l}19 \\
30 \\
39\end{array}$ & $\begin{array}{l}11 \\
13 \\
5.2\end{array}$ & $\begin{array}{r}.50 \\
.70 \\
1.4\end{array}$ & $\begin{array}{l}.90 \\
1.0 \\
.60\end{array}$ & $\begin{array}{r}93 \\
133 \\
118\end{array}$ & $\begin{array}{l}1.4 \\
1.8 \\
3.9\end{array}$ & $\begin{array}{l}2.7 \\
4.5 \\
6.7\end{array}$ & $\begin{array}{l}.460 \\
.510 \\
.290\end{array}$ & $\begin{array}{r}.050 \\
.010 \\
<.010\end{array}$ & $\begin{array}{l}127 \\
166 \\
153\end{array}$ \\
\hline $\begin{array}{l}3.16 \\
3.5\end{array}$ & $\begin{array}{l}4 / 12 / 84 \\
4 / 12 / 84\end{array}$ & $\begin{array}{l}41 \\
40\end{array}$ & $\begin{array}{l}13 \\
9.7\end{array}$ & $\begin{array}{l}2.5 \\
3.3\end{array}$ & $\begin{array}{l}4.5 \\
3.1\end{array}$ & $\begin{array}{l}143 \\
111\end{array}$ & $\begin{array}{l}2.7 \\
3.8\end{array}$ & $\begin{array}{l}34 \\
48\end{array}$ & $\begin{array}{r}.100 \\
<.100\end{array}$ & $\begin{array}{l}.030 \\
.080\end{array}$ & $\begin{array}{l}231 \\
204\end{array}$ \\
\hline 2.2 & $4 / 13 / 84$ & 26 & 7.9 & 1.6 & .80 & 96 & 3.1 & 6.5 & .240 & $<.010$ & 133 \\
\hline $\begin{array}{l}1.17 \\
1.9 \\
1.8 \\
1.5 \\
1.1\end{array}$ & $\begin{array}{l}4 / 12 / 84 \\
4 / 12 / 84 \\
4 / 12 / 84 \\
4 / 13 / 84 \\
4 / 12 / 84\end{array}$ & $\begin{array}{l}11 \\
18 \\
27 \\
27 \\
23\end{array}$ & $\begin{array}{l}6.3 \\
7.8 \\
11 \\
10 \\
8.8\end{array}$ & $\begin{array}{l}.70 \\
1.0 \\
1.4 \\
.60 \\
.90\end{array}$ & $\begin{array}{l}.90 \\
.80 \\
.60 \\
.50 \\
.60\end{array}$ & $\begin{array}{r}55 \\
78 \\
113 \\
111 \\
94\end{array}$ & $\begin{array}{l}1.9 \\
2.2 \\
4.5 \\
1.8 \\
2.1\end{array}$ & $\begin{array}{l}3.2 \\
3.7 \\
6.0 \\
5.9 \\
4.4\end{array}$ & $\begin{array}{l}<.100 \\
<.100 \\
<.100 \\
<.100 \\
<.100\end{array}$ & $\begin{array}{r}.010 \\
<.010 \\
<.010 \\
.080 \\
<.010\end{array}$ & $\begin{array}{r}85 \\
112 \\
151 \\
141 \\
121\end{array}$ \\
\hline 18.15 & $4 / 12 / 84$ & 130 & 20 & 5.7 & 3.0 & 358 & 16 & 87 & .320 & $<.010$ & 544 \\
\hline $\begin{array}{l}9.70 \\
9.77 \\
9.65 \\
9.63 \\
9.60 \\
9.52 \\
9.47 \\
9.46 \\
9.45 \\
9.18 \\
9.16\end{array}$ & $\begin{array}{l}4 / 13 / 84 \\
4 / 13 / 84 \\
4 / 13 / 84 \\
4 / 13 / 84 \\
4 / 13 / 84 \\
4 / 12 / 84 \\
4 / 12 / 84 \\
4 / 12 / 84 \\
4 / 12 / 84 \\
4 / 12 / 84 \\
4 / 12 / 84\end{array}$ & $\begin{array}{l}530 \\
9.8 \\
350 \\
270 \\
27 \\
81 \\
73 \\
45 \\
62 \\
41 \\
28\end{array}$ & $\begin{array}{l}75 \\
2.1 \\
39 \\
35 \\
16 \\
20 \\
12 \\
13 \\
13 \\
9.7 \\
9.0\end{array}$ & $\begin{array}{l}180 \\
2.8 \\
23 \\
39 \\
.60 \\
9.1 \\
9.9 \\
4.2 \\
7.1 \\
4.5 \\
2.0\end{array}$ & $\begin{array}{l}9.7 \\
1.2 \\
2.9 \\
4.4 \\
.40 \\
1.7 \\
2.1 \\
1.1 \\
1.6 \\
1.4 \\
.80\end{array}$ & $\begin{array}{r}314 \\
26 \\
26 \\
140 \\
139 \\
199 \\
107 \\
133 \\
120 \\
102 \\
112\end{array}$ & $\begin{array}{c}220 \\
3.5 \\
19 \\
47 \\
1.6 \\
20 \\
20 \\
9.9 \\
15 \\
10 \\
4.0\end{array}$ & $\begin{array}{c}190 \\
11 \\
9.2 \\
58 \\
1.1 \\
24 \\
25 \\
12 \\
19 \\
13 \\
3.2\end{array}$ & $\begin{array}{c}300 \\
3.90 \\
280 \\
210 \\
<.100 \\
20.0 \\
30.0 \\
9.90 \\
22.0 \\
9.20 \\
.150\end{array}$ & $\begin{array}{l}<.010 \\
<.010 \\
<.010 \\
<.010 \\
.020 \\
<.010 \\
<.010 \\
<.010 \\
<.010 \\
<.010 \\
<.010\end{array}$ & $\begin{array}{r}-- \\
-- \\
-- \\
-- \\
156 \\
414 \\
417 \\
232 \\
343 \\
210 \\
146\end{array}$ \\
\hline $\begin{array}{l}14.3 \\
14.5 \\
14.9\end{array}$ & $\begin{array}{l}4 / 12 / 84 \\
4 / 12 / 84 \\
4 / 12 / 84\end{array}$ & $\begin{array}{l}6.4 \\
5.0 \\
3.7\end{array}$ & $\begin{array}{l}1.9 \\
1.2 \\
1.4\end{array}$ & $\begin{array}{l}2.0 \\
1.5 \\
1.2\end{array}$ & $\begin{array}{l}1.8 \\
1.0 \\
2.3\end{array}$ & $\begin{array}{l}12 \\
13 \\
9.0\end{array}$ & $\begin{array}{l}1.2 \\
1.4 \\
1.3\end{array}$ & $\begin{array}{l}10 \\
11 \\
12\end{array}$ & $\begin{array}{l}<.100 \\
<.100 \\
<.100\end{array}$ & $\begin{array}{r}.050 \\
<.010 \\
<.010\end{array}$ & $\begin{array}{l}42 \\
-- \\
26\end{array}$ \\
\hline 11.2 & $4 / 12 / 84$ & 2.6 & 1.0 & 1.1 & .90 & 7.0 & 1.2 & 7.7 & $<.100$ & $<.010$ & 20 \\
\hline
\end{tabular}


Table 5.--Trace constituents and compounds

\begin{tabular}{|c|c|c|c|c|c|c|c|c|c|c|}
\hline $\begin{array}{l}\text { Si te } \\
\text { Number }\end{array}$ & $\begin{array}{c}\text { Date } \\
\text { of } \\
\text { sample }\end{array}$ & $\begin{array}{l}\text { Barium, } \\
\text { total } \\
\text { recov- } \\
\text { erable } \\
(\mu \mathrm{g} / \mathrm{L} \\
\text { as } \mathrm{Ba})\end{array}$ & $\begin{array}{l}\text { Beryl- } \\
\text { lium, } \\
\text { total } \\
\text { recov- } \\
\text { erable } \\
\text { ( } \mathrm{\mu g} / \mathrm{L} \\
\text { as } \mathrm{Be})\end{array}$ & $\begin{array}{l}\text { Cadmium } \\
\text { total } \\
\text { recov- } \\
\text { erable } \\
\text { ( } \mu \mathrm{g} / \mathrm{L} \\
\text { as Cd) }\end{array}$ & $\begin{array}{l}\text { Chro- } \\
\text { mium, } \\
\text { total } \\
\text { recov- } \\
\text { erable } \\
(\mu \mathrm{g} / \mathrm{L} \\
\text { as } \mathrm{Cr}) \\
\end{array}$ & $\begin{array}{l}\text { Copper, } \\
\text { total } \\
\text { recov- } \\
\text { erable } \\
(\mu \mathrm{g} / \mathrm{L} \\
\text { as } \mathrm{Cu})\end{array}$ & $\begin{array}{c}\text { Ganide } \\
\text { total } \\
\text { (mg/L } \\
\text { as CN) }\end{array}$ & $\begin{array}{l}\text { Iron, } \\
\text { total } \\
\text { recov- } \\
\text { erable } \\
(\mu \mathrm{g} / \mathrm{L} \\
\text { as } \mathrm{Fe}) \\
\end{array}$ & $\begin{array}{l}\text { Lead, } \\
\text { total } \\
\text { recov- } \\
\text { erable } \\
\text { ( } \mathrm{g} / \mathrm{L} \\
\text { as } \mathrm{Pb}) \\
\end{array}$ & $\begin{array}{l}\text { Li thium } \\
\text { total } \\
\text { recov- } \\
\text { erable } \\
\text { ( } \mathrm{\mu g} / \mathrm{L} \\
\text { as Li) } \\
\end{array}$ \\
\hline 8.0 & $4 / 12 / 84$ & $<100$ & $<10$ & $<1$ & 3 & 2 & $<.01$ & 200 & 1 & 10 \\
\hline 7.23 & $\begin{array}{l}4 / 12 / 84 \\
6 / 3 / 84\end{array}$ & $<100$ & $<10$ & $<1$ & 2 & 1 & $<.01$ & 710 & 1 & $<10$ \\
\hline 7.19 & $\begin{array}{l}4 / 12 / 84 \\
6 / 3 / 84\end{array}$ & 200 & $<10$ & 1 & 3 & 4 & $<.01$ & 180 & 4 & 10 \\
\hline 7.30 & $4 / 12 / 84$ & $<100$ & $<10$ & $<1$ & 1 & 1 & $<.01$ & 230 & 1 & $<10$ \\
\hline 7.18 & $\begin{array}{l}4 / 12 / 84 \\
6 / 3 / 84\end{array}$ & 100 & $<10$ & $<1$ & $\begin{array}{r}3 \\
--\end{array}$ & 2 & $<.01$ & 190 & 3 & 10 \\
\hline & $4 / 12 / 84$ & $<100$ & $<10$ & $<1$ & 3 & 1 & $<.01$ & 100 & 2 & 10 \\
\hline 7.12 & $4 / 12 / 84$ & $<100$ & $<10$ & $<1$ & 2 & 1 & $<.01$ & 170 & 1 & $<10$ \\
\hline \multirow{3}{*}{$\begin{array}{l}5.14 \\
5.9 \\
5.5\end{array}$} & $4 / 12 / 84$ & $<100$ & $<10$ & $<1$ & $\begin{array}{l}3 \\
3\end{array}$ & 1 & $<.01$ & 210 & 3 & $<10$ \\
\hline & $4 / 12 / 84$ & $<100$ & $<10$ & $\begin{array}{l}<1 \\
<1\end{array}$ & 3 & 2 & $<.01$ & 120 & 5 & 20 \\
\hline & $4 / 13 / 84$ & $<100$ & $<10$ & & $<1$ & 1 & $<.01$ & 190 & 2 & $<10$ \\
\hline \multirow{2}{*}{$\begin{array}{l}3.16 \\
3.5\end{array}$} & $4 / 12 / 84$ & 100 & $<10$ & $<1$ & 3 & 1 & $<.01$ & 1600 & 5 & 90 \\
\hline & $4 / 12 / 84$ & 100 & $<10$ & $<1$ & 7 & 4 & $<.01$ & 290 & 5 & 90 \\
\hline 2.2 & $\begin{array}{l}4 / 13 / 84 \\
6 / 3 / 84\end{array}$ & $<100$ & $\begin{array}{l}<10 \\
--\end{array}$ & $\begin{array}{l}<1 \\
--\end{array}$ & 1 & $\begin{array}{r}2 \\
--\end{array}$ & $<.01$ & 250 & 1 & 10 \\
\hline \multirow{5}{*}{$\begin{array}{l}1.17 \\
1.9 \\
1.8 \\
1.5 \\
1.1\end{array}$} & $4 / 12 / 8$ & $<100$ & $<10$ & $<1$ & 2 & 1 & $<.01$ & 130 & 1 & $<10$ \\
\hline & $4 / 12 / 84$ & $<100$ & $<10$ & 3 & 4 & 3 & $<.01$ & 300 & 4 & 10 \\
\hline & $4 / 12 / 84$ & $<100$ & $<10$ & $<1$ & 4 & 1 & $<.01$ & 180 & 2 & 10 \\
\hline & $4 / 12 / 84$ & $<100$ & $<10$ & $<1$ & 2 & 2 & $<.01$ & 90 & 3 & $<10$ \\
\hline & $4 / 12 / 84$ & $<100$ & $<10$ & $<1$ & 2 & 1 & $<.01$ & 220 & 1 & $<10$ \\
\hline \multirow[t]{2}{*}{18.15} & $4 / 12 / 84$ & 500 & $<10$ & 1 & 12 & 29 & $<.01$ & 31000 & 73 & 20 \\
\hline & & & & & & & & & & \\
\hline 9.70 & $\begin{array}{l}4 / 13 / 84 \\
6 / 3 / 84\end{array}$ & $\begin{array}{r}<100 \\
--\end{array}$ & $<10$ & $\begin{array}{l}<1 \\
--\end{array}$ & 30 & $\begin{array}{r}2 \\
--\end{array}$ & $<.01$ & $\begin{array}{r}740 \\
---\end{array}$ & $\begin{array}{r}6 \\
--\end{array}$ & $<10$ \\
\hline \multirow[t]{2}{*}{9.77} & $\begin{array}{l}4 / 13 / 84 \\
6 / 3 / 84\end{array}$ & $<100$ & $<10$ & $\begin{array}{l}<1 \\
--\end{array}$ & 4 & 2 & $<.01$ & 740 & 6 & $<10$ \\
\hline & & 2400 & $<-\overline{10}$ & 58 & 1 & 2 & .06 & 240 & $\overline{2}$ & - \\
\hline \multirow{2}{*}{9.63} & $\begin{array}{l}4 / 13 / 84 \\
4 / 13 / 84\end{array}$ & $\begin{array}{r}2400 \\
800\end{array}$ & $<10$ & 27 & 4 & 2 & .06 & 120 & $\begin{array}{l}2 \\
4\end{array}$ & $\begin{array}{r}10 \\
20\end{array}$ \\
\hline & & & & -- & -- & -- & -- & -. & - & -- \\
\hline 9.60 & $4 / 13 / 84$ & 100 & $<10$ & $<1$ & 2 & 1 & $<.01$ & 100 & 2 & $<10$ \\
\hline & $4 / 12 / 84$ & 100 & $<10$ & $<1$ & 2 & 2 & $<.01$ & 150 & 1 & 20 \\
\hline \multirow[t]{2}{*}{9.47} & $4 / 12 / 84$ & 200 & $<10$ & 1 & 1 & 2 & .01 & 480 & 1 & 350 \\
\hline & & - 100 & $-\overline{10}$ & - & $=$ & - & -- & -- & -- & -- \\
\hline 9.46 & $4 / 12 / 84$ & $\begin{array}{r}<100 \\
200\end{array}$ & $\begin{array}{l}<10 \\
<10\end{array}$ & $<1$ & 2 & 1 & $<.01$ & 100 & 2 & 20 \\
\hline 9.45 & $4 / 12 / 84$ & 200 & $<10$ & 1 & 2 & 2 & $<.01$ & 210 & 1 & 210 \\
\hline \multirow[t]{2}{*}{9.18} & $4 / 12 / 84$ & 100 & $<10$ & $<1$ & 2 & 3 & $<.01$ & 240 & 1 & 90 \\
\hline & & & & -- & - & -- & -- & -- & - & -- \\
\hline 9.16 & $4 / 12 / 84$ & $<100$ & $<10$ & $<1$ & 3 & 2 & $<.01$ & 360 & 5 & 10 \\
\hline 14.3 & $\begin{array}{l}4 / 12 / 84 \\
6 / 3 / 84\end{array}$ & $<100$ & $<10$ & $\begin{array}{l}<1 \\
--\end{array}$ & 1 & 2 & $<.01$ & 410 & 2 & $<10$ \\
\hline 14.5 & $4 / 12 / 84$ & $<100$ & $<10$ & $<1$ & 5 & 3 & $<.01$ & 940 & 4 & 10 \\
\hline 14.9 & $4 / 2 / 84$ & $<100$ & $<10$ & $<1$ & 3 & 2 & $<.01$ & 270 & 3 & $<10$ \\
\hline 11.2 & $4 / 12 / 84$ & $<100$ & $<10$ & $<1$ & 1 & 2 & $<.01$ & 570 & 2 & $<10$ \\
\hline
\end{tabular}


Table 5.--Trace constituents and compounds--Continued

\begin{tabular}{|c|c|c|c|c|c|c|c|c|c|c|c|}
\hline $\begin{array}{l}\text { Manga- } \\
\text { nese, } \\
\text { total } \\
\text { recov- } \\
\text { erable } \\
(\mu \mathrm{g} / \mathrm{L} \\
\text { as } \mathrm{Mn}) \\
\end{array}$ & $\begin{array}{l}\text { Mercury } \\
\text { total } \\
\text { recov- } \\
\text { erable } \\
(\mu \mathrm{g} / \mathrm{L} \\
\text { as } \mathrm{Hg})\end{array}$ & $\begin{array}{l}\text { Molyb- } \\
\text { denum, } \\
\text { total } \\
\text { recov- } \\
\text { erable } \\
\text { ( } \mu \mathrm{g} / \mathrm{L} \\
\text { as Mo) } \\
\end{array}$ & $\begin{array}{l}\text { Stron- } \\
\text { tium, } \\
\text { total } \\
\text { recov- } \\
\text { erable } \\
\text { ( } \mathrm{Hg} / \mathrm{L} \\
\text { as Sr) } \\
\end{array}$ & $\begin{array}{l}\text { Uranium } \\
\text { dis- } \\
\text { solved, } \\
\text { extrac- } \\
\text { tion } \\
(\mu \mathrm{g} / \mathrm{L})\end{array}$ & $\begin{array}{c}\text { Uranium } \\
\text { natural } \\
\text { dis- } \\
\text { solved } \\
(\mu \mathrm{g} / \mathrm{L} \\
\text { as U) }\end{array}$ & $\begin{array}{l}\text { Vana- } \\
\text { dium, } \\
\text { di s- } \\
\text { solved } \\
\text { ( } \mu \mathrm{g} / \mathrm{L} \\
\text { as V) } \\
\end{array}$ & $\begin{array}{l}\text { Zinc, } \\
\text { totai } \\
\text { recov- } \\
\text { erable } \\
\text { ( } \mu \mathrm{g} / \mathrm{L} \\
\text { as } \mathrm{Zn}) \\
\end{array}$ & $\begin{array}{l}\text { Carbon } \\
\text { organic } \\
\text { total } \\
\text { (mg/L) } \\
\text { as C) }\end{array}$ & $\begin{array}{c}\text { Carbon, } \\
\text { organic } \\
\text { dis- } \\
\text { solved } \\
(\mathrm{mg} / \mathrm{L} \\
\text { as C) }\end{array}$ & $\begin{array}{l}\text { Oil and } \\
\text { grease, } \\
\text { total } \\
\text { recov. } \\
\text { gravi- } \\
\text { metric } \\
\text { (mg/L) }\end{array}$ & $\begin{array}{l}\text { Cobalt, } \\
\text { total } \\
\text { recov- } \\
\text { erable } \\
(\mu \mathrm{g} / \mathrm{L} \\
\text { as Co) } \\
\end{array}$ \\
\hline 10 & $<.1$ & 3 & 260 & - & 1.1 & $<1$ & 10 & 1.3 & - & -- & $<1$ \\
\hline 30 & $<.1$ & $<1$ & 20 & .08 & -- & 1 & 20 & 2.0 & - & $\cdots$ & 1 \\
\hline-- & - & -- & -- & - & -- & $\therefore$ & -- & $\cdots$ & 15 & - & - \\
\hline 430 & .1 & $<1$ & 140 & $\cdots$ & .8 & $<1$ & 10 & 5.1 & -- & - & 7 \\
\hline -- & - & -- & -. & $\cdots$ & - & -. & - & $\cdots$ & 10 & $<1$ & - \\
\hline 30 & $<.1$ & $<1$ & 110 & - & .9 & $<1$ & 10 & .90 & -- & $\cdots$ & 1 \\
\hline 20 & $<.1$ & $<1$ & 100 & - & 1.0 & $<1$ & 10 & 1.4 & -- & - & $<1$ \\
\hline$\cdots$ & $\cdots$ & $\cdots$ & - & - & - & $\cdots$ & - & $\cdots$ & 2.0 & - & $\ldots$ \\
\hline 20 & $<.1$ & $<1$ & 30 & - & .9 & 1 & 10 & .60 & $\cdots$ & - & 5 \\
\hline$<10$ & $<.1$ & $<1$ & 10 & - & .7 & $<1$ & 30 & .70 & -- & - & $<1$ \\
\hline 20 & $<.1$ & $<1$ & 20 & -- & .7 & $<1$ & 40 & .60 & -- & -- & 2 \\
\hline 20 & $<.1$ & $<1$ & 40 & -- & 1.4 & $<1$ & 20 & .80 & -- & - & 3 \\
\hline 10 & .1 & $<1$ & 50 & .11 & -. & $<1$ & 30 & .70 & - & -. & 1 \\
\hline 1000 & $<.1$ & 11 & 770 & -- & 1.0 & $<1$ & 20 & .40 & - & - & 10 \\
\hline 50 & $<.1$ & 32 & 230 & $\cdots$ & 2.5 & 30 & 20 & .70 & -- & -- & 1 \\
\hline 10 & .2 & 1 & 30 & $\cdots$ & 1.0 & $<1$ & 20 & 1.9 & - & $\cdots$ & $<1$ \\
\hline-- & -- & -- & - & - & $\cdots$ & -- & -- & $\cdots$ & 4.4 & $\cdots$ & -- \\
\hline 10 & $<.1$ & $<1$ & 10 & .08 & - & - & 10 & .80 & - & -- & $<1$ \\
\hline 10 & $<.1$ & $<1$ & 20 & - & .8 & $<1$ & 30 & .60 & - & - & 6 \\
\hline 10 & .1 & $<1$ & 30 & - & .9 & $<1$ & 20 & .50 & -- & -- & 5 \\
\hline$<10$ & $<.1$ & 1 & 40 & .17 & - & - & 60 & .60 & - & - & $<1$ \\
\hline 10 & $<.1$ & $<1$ & 20 & -- & .8 & 1 & 10 & .70 & -- & - & 1 \\
\hline 11000 & 4.5 & $<1$ & 250 & -- & 16 & $<1$ & 90 & 21 & - & -- & 30 \\
\hline - & - & - & - & - & - & - & -- & - & 4.1 & 4 & - \\
\hline 80 & $<.1$ & $<1$ & 30 & -. & 1300 & $<1$ & 20 & 2.2 & - & - & - \\
\hline - & -- & - & - & -- & $\cdots$ & -- & -- & $\cdots$ & 3.2 & $\cdots$ & - \\
\hline 80 & $<.1$ & $<1$ & 30 & .40 & -- & $<1$ & 20 & 2.3 & - & -- & 3 \\
\hline- & -- & -- & -- & -- & $\cdots$ & - & -- & $\cdots$ & 5.0 & - & $\cdots$ \\
\hline 5900 & .1 & $<1$ & 1100 & 1.0 & - & $<1$ & 40 & 2.3 & $\cdots$ & -- & 20 \\
\hline 2900 & .1 & $<1$ & 720 & -. & 190 & $<1$ & 30 & 2.7 & -- & -- & 10 \\
\hline-- & -- & - & - & - & $\cdots$ & -- & -- & $\cdots$ & 1.3 & $<1$ & -- \\
\hline$<10$ & $<.1$ & $<1$ & 20 & -- & .8 & $<1$ & 10 & .50 & -- & -- & $<1$ \\
\hline 40 & .2 & $<1$ & 120 & - & 110 & $<1$ & 20 & .90 & - & - & 4 \\
\hline 430 & .2 & 1 & 210 & - & 180 & $<1$ & 10 & 2.0 & - & -- & $<1$ \\
\hline-- & - & - & -- & - & $\cdots$ & - & - & $\cdots$ & 3.4 & - & - \\
\hline 10 & .2 & 1 & 70 & -- & 61 & $<1$ & 50 & .80 & $\cdots$ & - & $<1$ \\
\hline 250 & .2 & $<1$ & 120 & - & 140 & $<1$ & 30 & 1.5 & - & -- & 6 \\
\hline 20 & $<.1$ & $<1$ & 60 & -- & 68 & $<1$ & 20 & 1.1 & - & - & 1 \\
\hline-- & -- & - & $\cdots$ & - & - & $\cdots$ & -- & $\cdots$ & 2.5 & - & - \\
\hline 10 & .1 & $<1$ & 30 & .23 & -- & $<1$ & 20 & .80 & - & -- & 2 \\
\hline 10 & $<.1$ & 1 & 20 & .02 & - & $<1$ & 20 & 1.5 & - & -- & $<1$ \\
\hline$\cdots$ & - & $\therefore$ & $\cdots$ & - & -- & $\cdots$ & -- & $\cdots$ & 1.7 & $<1$ & -- \\
\hline 20 & $<.1$ & $<1$ & 20 & .05 & -. & $<1$ & 20 & 1.4 & $\cdots$ & - & 3 \\
\hline 10 & $<.1$ & $<1$ & 20 & .01 & - & $<1$ & 20 & 1.1 & - & -- & 4 \\
\hline 30 & $<.1$ & $<1$ & -- & $<.01$ & -. & 1 & 20 & 1.3 & - & - & $<1$ \\
\hline
\end{tabular}


Table 6. --Radiochemicals

\begin{tabular}{|c|c|c|c|c|c|c|c|}
\hline $\begin{array}{c}\text { Site } \\
\text { number }\end{array}$ & $\begin{array}{l}\text { Date } \\
\text { of } \\
\text { sample }\end{array}$ & $\begin{array}{l}\text { Gross } \\
\text { a lpha, } \\
\text { dis- } \\
\text { solved } \\
\text { ( } \mu \mathrm{g} / \mathrm{L} \\
\quad \text { as } \\
\text { U-NAT) }\end{array}$ & $\begin{array}{c}\text { Gross } \\
\text { alpha, } \\
\text { susp. } \\
\text { total } \\
\text { ( } \mu \mathrm{g} / \mathrm{L} \\
\text { as } \\
\text { U-NAT) }\end{array}$ & $\begin{array}{c}\text { Gross } \\
\text { beta, } \\
\text { dis- } \\
\text { solved } \\
\text { (pCi/L) } \\
\text { as } \\
\text { Cs-137) }\end{array}$ & $\begin{array}{c}\text { Gross } \\
\text { beta, } \\
\text { dis- } \\
\text { solved } \\
(\mathrm{pCi} / \mathrm{L}) \\
\text { as } \mathrm{Sr} / \\
\mathrm{Y}-90)\end{array}$ & $\begin{array}{c}\text { Gross } \\
\text { beta, } \\
\text { susp. } \\
\text { total } \\
\text { (pCi/L } \\
\text { as } \\
\text { Cs-137) }\end{array}$ & $\begin{array}{l}\text { Gross } \\
\text { beta, } \\
\text { susp. } \\
\text { total } \\
(\mathrm{pCi} / \mathrm{L}) \\
\text { as } \mathrm{Sr} / \\
\mathrm{Y}-90) \\
\end{array}$ \\
\hline 7.30 & $4 / 12 / 84$ & $<5.0$ & 0.6 & 3.1 & $<2.7$ & 0.7 & 0.7 \\
\hline 5.14 & $4 / 12 / 84$ & $<2.3$ & .9 & $<1.4$ & $<1.2$ & .8 & .7 \\
\hline 3.5 & $4 / 12 / 84$ & $<4.6$ & 2.4 & $<2.6$ & $<2.2$ & 1.9 & 1.7 \\
\hline 2.2 & $4 / 13 / 84$ & 2.6 & $<.4$ & 1.3 & 1.2 & $<.5$ & $<.4$ \\
\hline 18.15 & $4 / 12 / 84$ & $<13$ & 39 & 7.1 & 6.1 & 29 & 25 \\
\hline $\begin{array}{l}9.63 \\
9.60 \\
9.47 \\
9.46\end{array}$ & $\begin{array}{l}4 / 13 / 84 \\
4 / 13 / 84 \\
4 / 12 / 84 \\
4 / 12 / 84\end{array}$ & $\begin{array}{c}160 \\
<3.5 \\
94 \\
20\end{array}$ & $\begin{array}{r}10 \\
1.2 \\
3.7 \\
1.2\end{array}$ & $\begin{array}{l}320 \\
<1.8 \\
61 \\
19\end{array}$ & $\begin{array}{l}280 \\
<1.6 \\
53 \\
16\end{array}$ & $\begin{array}{l}49 \\
37^{11}\end{array}$ & $\begin{array}{l}44 \\
34^{.8} \\
9.9\end{array}$ \\
\hline 14.3 & $4 / 12 / 84$ & $<.9$ & $<.4$ & 2.2 & 1.9 & $<.4$ & $<.4$ \\
\hline
\end{tabular}




\section{REFERENCES}

Evaldi, R. D., 1984, Streamflow and specific conductance data for selected sites, February 15 through April 9, 1984, near the Y-12 Plant, the Oak Ridge Reservation, Tennessee: U.S. Geological Survey Open-File Report 84-625, 5 p.

Goerlitz, D. F., and Brown, Eugene, 1972 , Methods for analysis of organic substances in water: U.S. Geological Survey Techniques of Water-Resources Investigations, Book 5, Chap. A3, $40 \mathrm{p}$.

McMaster, W. M., 1967, Hydrologic data for the Oak Ridge area Tennessee: U.S. Geological Survey Water-Supply Paper 1839-N, $60 \mathrm{p}$.

Miller, R. A., 1974, The geologic history of Tennessee: Tennessee Division of Geology Bulletin 74,63 p.

Skougstad, M. W., Fishman, M. J., Friedman, L. C., Erdmann, D. E., and Duncan, S. S., eds., 1979, Methods for determination of inorganic substances in water and fluvial sediments: U.S. Geological Survey Techniques of Water-Resources Investigations, Book 5, Chap. A1, 626 p.

Thacker, L. L., Janzer, V. J., and Edwards, K. W., 1977, Methods for determination of radioactive substances in water and fluvial sediments: U.S. Geological Survey Techniques of Water-Resources Investigations, Book 5, Chap. A5, 95 p. 


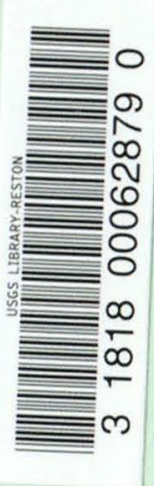

